\title{
In Vitro Evaluation of Positive Expiratory Pressure Devices Attached to Nebulizers
}

\author{
Ariel Berlinski MD
}

\begin{abstract}
BACKGROUND: Patients with cystic fibrosis perform airway clearance techniques and receive nebulized medications on a regular basis. Some positive expiratory pressure (PEP) devices allow concomitant administration of aerosol. I hypothesized that this practice alters the aerosol characteristics and patient dose. I compared the aerosol characteristics and patient dose of nebulized albuterol from 2 types of nebulizer, alone and when connected to different PEP and vibratory PEP devices. METHODS: Three units of a continuous-output nebulizer (Up-Draft II Opti-Neb) and 3 units of a breath-enhanced nebulizer (LC Plus) were tested alone and connected to PEP devices (Acapella Choice, Acapella Duet, and EzPAP for Up-Draft II Opti-Neb, and Pari PEP at 2 different settings, and Pari PEP S system with the LC Plus). Aerosol characteristics were evaluated with a cooled cascade impaction technique. The nebulizers were loaded with $2.5 \mathrm{mg} / 3 \mathrm{~mL}$ albuterol solution and operated for $4 \mathrm{~min}$ at $6 \mathrm{~L} / \mathrm{min}$ (wall air). Patient dose was evaluated with simulated breathing patterns for a child, small adult, and large adult. Albuterol was assayed via spectrophotometry. RESULTS: Connecting the LC Plus to the PEP devices did not change the aerosol characteristics or patient dose. Connecting the Up-Draft II Opti-Neb to the PEP devices significantly reduced the mass median aerodynamic diameter, from $4.13 \mu \mathrm{m}$ to $3.72 \mu \mathrm{m}$ with $\operatorname{EzPAP}(P=.02)$, $1.24 \mu \mathrm{m}$ with Acapella Choice $(P<.001)$, and $1.22 \mu \mathrm{m}$ with Acapella Duet $(P<.001)$. The total amount of albuterol captured by the impactor decreased when connected to either Acapella Choice (65\%) or Acapella Duet (69\%), with 17-25\% retained in the PEP devices. Patient dose decreased by $76 \%$ and $84 \%$ when connected to Acapella Choice and Acapella Duet, respectively. CONCLUSIONS: Concomitant use of nebulizer and PEP or vibratory PEP devices that obstruct the aerosol pathway significantly decrease the aerosol particle size and the patient dose. Key words: PEP; vibratory PEP; nebulizer; particle size; aerosol characteristics; albuterol. [Respir Care 2014;59(2):216-222. (C) 2014 Daedalus Enterprises]
\end{abstract}

\section{Introduction}

Cystic fibrosis is the most common genetic lethal disease in the white population. A defect in the cystic fibrosis

Dr Berlinski is affiliated with the Pulmonology Section, Department of Pediatrics, University of Arkansas for Medical Sciences, and with the Pediatric Aerosol Research Laboratory, Arkansas Children's Hospital Research Institute, Little Rock, Arkansas.

Dr Berlinski presented a version of this paper at the North American Cystic Fibrosis Conference, held October 21-23, 2010, in Baltimore, Maryland.

This study was partly supported by Grant 036072 from the University of Arkansas for Medical Sciences College of Medicine Children's University Medical Group Fund Grant Program. The Pediatric Aerosol Research Laboratory at Arkansas Children's Hospital Research Institute was par- transmembrane regulator channel leads to a multi-organ disease that mainly affects the lungs and nutritional status. ${ }^{1}$ Currently recommended therapies include several aerosols (dornase alfa, 7\% hypertonic saline, albuterol,

\footnotetext{
tially established by and receives partial support from the George Endowment for Asthma. Dr Berlinski has disclosed relationships with Johnson \& Johnson, MPEX Pharmaceutical, Gilead, Philips, Genentech, Vertex, Abvie, and S\&T Technologies. None of their products are discussed in this paper.

Correspondence: Ariel Berlinski MD, Pulmonary Medicine, Department of Pediatrics, University of Arkansas for Medical Sciences, 1 Children's Way, Slot 512-17, Little Rock AR 72202. E-mail: Berlinski Ariel@uams.edu.
}

DOI: $10.4187 /$ respcare. 02698 
antibiotics, and corticosteroids), airway clearance therapies, nutritional support, and enzyme and vitamin replacement therapy. ${ }^{2,3}$

The addition of new therapies has increased the amount of time patients with cystic fibrosis devote to their treatments. Aerosol treatments and airway clearance therapies are combined to try to reduce treatment time. Aerosols are administered during high-frequency chest compression, without changing the configuration of the nebulizer. Positive expiratory pressure (PEP) and vibratory PEP devices can be adapted to concomitantly administer the aerosol and perform the airway clearance therapies. ${ }^{4}$

Particle size is an important factor in determining intrapulmonary deposition of inhaled aerosols. ${ }^{5}$ Laube et al reported a decrease in intrapulmonary deposition and a slightly more peripheral aerosol distribution when a breath-enhanced nebulizer was coupled to a PEP device. ${ }^{6}$ Dornelas de Andrade et al reported a decrease in intrapulmonary deposition when a vibratory PEP device was coupled to a nebulizer. ${ }^{7}$ The coupling of nebulizer and $\mathrm{PEP} /$ vibratory PEP could result in intra-device aerosol impaction, thus affecting particle size and amount and distribution of intrapulmonary deposition.

I hypothesized that the concomitant use of PEP/ vibratory PEP devices connected to a nebulizer would change the aerosol characteristics of nebulized albuterol. In this in vitro study I compared aerosol characteristics and patient dose with a continuous-output nebulizer and a breath-enhanced nebulizer, alone and when connected to the PEP/vibratory PEP devices.

\section{Methods}

This in vitro study was performed at the Pediatric Aerosol Research Laboratory at the Arkansas Children's Hospital Research Institute. The study included 2 parts: determination of particle size via cascade impaction, and determination of albuterol output via breathing simulation.

\section{Nebulizers and PEP Devices}

Three units of a continuous-output jet nebulizer (UpDraft II Opti-Neb, Teleflex Medical, Research Triangle Park, North Carolina) and 3 units of a breath-enhanced nebulizer (LC Plus, Pari Respiratory Equipment, Midlothian, Virginia) (LC Plus) were tested. Figure 1 shows the setups. The Up-Draft II Opti-Neb was tested with the Acapella Choice, Acapella Duet, and EzPAP PEP devices (all by Smiths Medical, Watford, United Kingdom). With the Acapella Choice and Acapella Duet the expiratory resistance setting was 1, and with the EzPAP the pressure was $5 \mathrm{~cm} \mathrm{H}_{2} \mathrm{O}$.

The Acapella Duet is a transparent reusable vibratory PEP device that allows concomitant nebulization when

\section{QUICK LOOK}

\section{Current knowledge}

Airway-clearance therapy for patients with cystic fibrosis commonly includes aerosol and positive expiratory pressure (PEP) or vibratory PEP devices. Devices that combine aerosol therapy and PEP have been introduced to enhance aerosol therapy and reduce airway-clearance treatment duration.

\section{What this paper contributes to our knowledge}

Simultaneous administration of nebulized aerosol with PEP (or vibratory PEP) devices that obstruct the aerosol pathway significantly reduced the aerosol particle size and the dose delivered to the patient.

placing the nebulizer at the port located at the bottom of the device. Corrugated tubing was placed at the end of the device to act as an aerosol reservoir. The Acapella Duet has a one-way inspiratory valve. The Acapella Choice is a green reusable vibratory PEP device that allows concomitant nebulization when connecting the nebulizer at the distal fitting of the device via T-piece. The EzPAP is a transparent PEP device that allows concomitant nebulization by placing a T-piece with nebulizer between the PEP device and the mouthpiece.

The LC Plus was tested alone, with the Pari PEP system (Pari Respiratory Equipment, Midlothian, Virginia, with resistance settings of 1.5 and 4.5), and with the Pari PEP S system (with resistance setting of 1.5) (Fig. 2). All connections were made according to manufacturer recommendations. The Pari PEP system replaces the nebulizer inspiratory valve of the Pari LC Plus, and it is used with a mouthpiece, without exhalation valve. The Pari PEP S connects to the Pari LC Plus, replacing the mouthpiece, and has different resistance settings, providing a pressure range between 10 and $20 \mathrm{~cm} \mathrm{H}_{2} \mathrm{O}$.

\section{Aerosol Particle Size Characterization}

The nebulizers were weighed on a precision scale when dry, and after loading a unit dose $(2.5 \mathrm{mg} / 3 \mathrm{~mL})$ of albuterol sulfate (Dey, Napa, California). The nebulizers were operated at $6 \mathrm{~L} / \mathrm{min}$ with wall air, and the accuracy of the flow was verified before each test with a mass flow meter (4043, TSI, Shoreview, Minnesota). The nebulizers were then connected to a cascade impactor (Next Generation Impactor, MSP, Shoreview, Minnesota) assembled with internal and external filters (GC-50, Advantec MFS, Dublin, California) cooled to $4{ }^{\circ} \mathrm{C}$ for $90 \mathrm{~min}$. The cascade impactor was connected to a suction pump (HCP5, Copley 


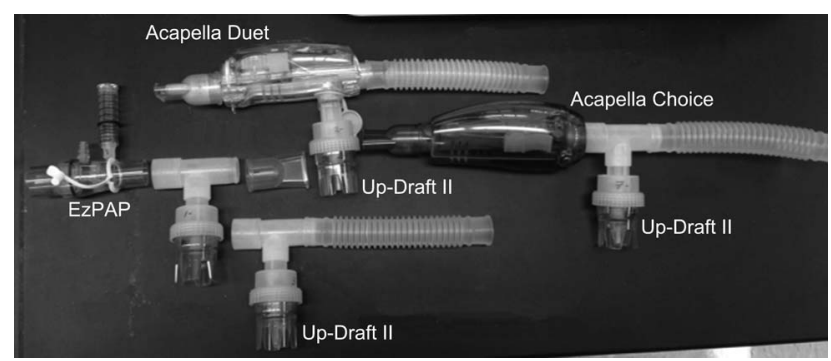

Fig. 1. Test setups with the Up-Draft II Opti-Neb nebulizer and 3 positive expiratory pressure (PEP) devices: Acapella Duet, Acapella Choice, and EzPAP. The extension tube length is $15 \mathrm{~cm}$.

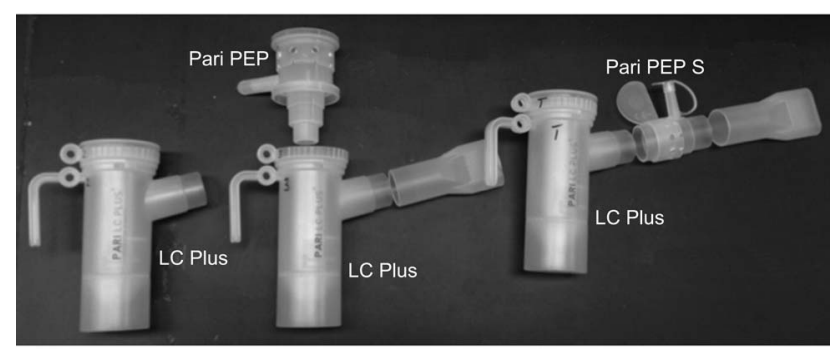

Fig. 2. Test setups with the LC Plus nebulizer and 2 positive expiratory pressure (PEP) devices: Pari PEP, and Pari PEP S.

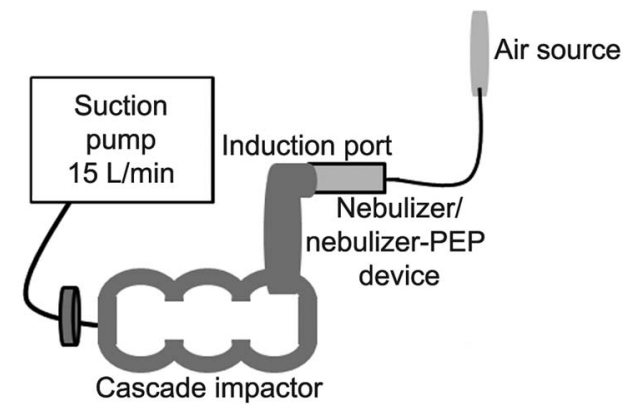

Fig. 3. Experimental setup to measure aerosol characteristics.

Scientific, Nottingham, United Kingdom) calibrated to $15 \mathrm{~L} / \mathrm{min}$ with a mass flow meter (4043, TSI, Shoreview, Minnesota), and used within $5 \mathrm{~min}$ of removing it from the refrigerator. ${ }^{8}$ The Up-Draft II Opti-Neb was adapted to the impactor's induction port with a T-piece. The LC Plus was connected by its mouthpiece with its exhalation port sealed (Fig. 3). The nebulizers were operated for $4 \mathrm{~min}$, after which the nebulizers were re-weighed, and the cascade impactor was disassembled. They were all washed with ultra-pure water, which was analyzed for albuterol via spectrophotometry (Biomate $3 \mathrm{UV}-\mathrm{Vis}$, Thermo Fisher Scientific, Waltham, Massachusetts), at $276 \mathrm{~nm}$. Samples with a known albuterol concentration were used to create the calibration curve. The Acapella Choice and Acapella Duet devices were also washed with ultra-pure water, which was analyzed for albuterol concentration.

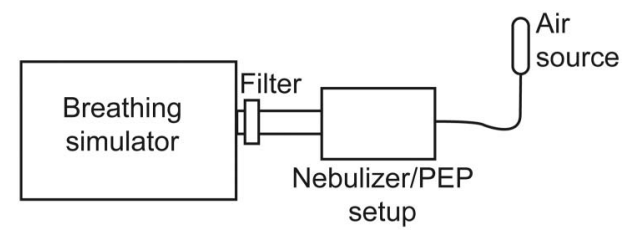

Fig. 4. Experimental setup to measure patient dose with breathing simulation.

The nebulizers, either alone or connected to the PEP devices, were tested in duplicate $(n=6$ for each configuration).

The following parameters were calculated according to the United States Pharmacopeia and European Pharmacopeia recommendations, using CITDAS V3.1 software (Copley Scientific, Nottingham, United Kingdom): mass median aerodynamic diameter (MMAD), geometric standard deviation, percentage of particles $<5 \mu \mathrm{m}$, percentage of particles $1-3 \mu \mathrm{m},{ }^{8-10}$ and total albuterol mass captured by the cascade impactor. Mass balance was calculated for each device.

\section{Patient Dose}

Patient dose was evaluated with a breathing simulation technique. Three units of the Up-Draft II Opti-Neb and LC Plus were tested. The nebulizer was weighed dry, and after $2.5 \mathrm{mg} / 3 \mathrm{~mL}$ of albuterol sulfate was loaded in the nebulizer. The nebulizers were operated for $5 \mathrm{~min}$ at $6 \mathrm{~L} /$ min with wall air, and the accuracy of the flow was verified before each test with a mass flow meter (4043, TSI, Shoreview, Minnesota). The nebulizers were then connected to a low-dead-space filter holder containing an inhalation filter (Pari Respiratory Equipment, Midlothian, Virginia) and connected in series to a breathing simulator (Compass, Pari Respiratory Equipment, Midlothian, Virginia) (Fig. 4). Three breathing patterns were tested:

- Older child: tidal volume $\left(\mathrm{V}_{\mathrm{T}}\right) 200 \mathrm{~mL}$, breathing frequency 20 breaths/min, inspiratory/expiratory ratio 1:2

- Small adult: $\mathrm{V}_{\mathrm{T}} 500 \mathrm{~mL}$, breathing frequency 15 breaths/ min, inspiratory/expiratory ratio $1: 2$

- Large adult: $\mathrm{V}_{\mathrm{T}} 770 \mathrm{~mL}$, breathing frequency 12 breaths/ min, inspiratory/expiratory ratio $1: 2$

The accuracy of the delivered $\mathrm{V}_{\mathrm{T}}$ was verified with a mass flow meter (4043, TSI, Shoreview, Minnesota). The nebulizers were reweighed upon completion of testing. The filters were washed with ultra-pure water. The filters and the nebulizer cups were analyzed for albuterol concentration via spectrophotometry.

The nebulizers were tested alone and connected to the PEP devices, using each of the breathing patterns $(n=3$ for each configuration). 


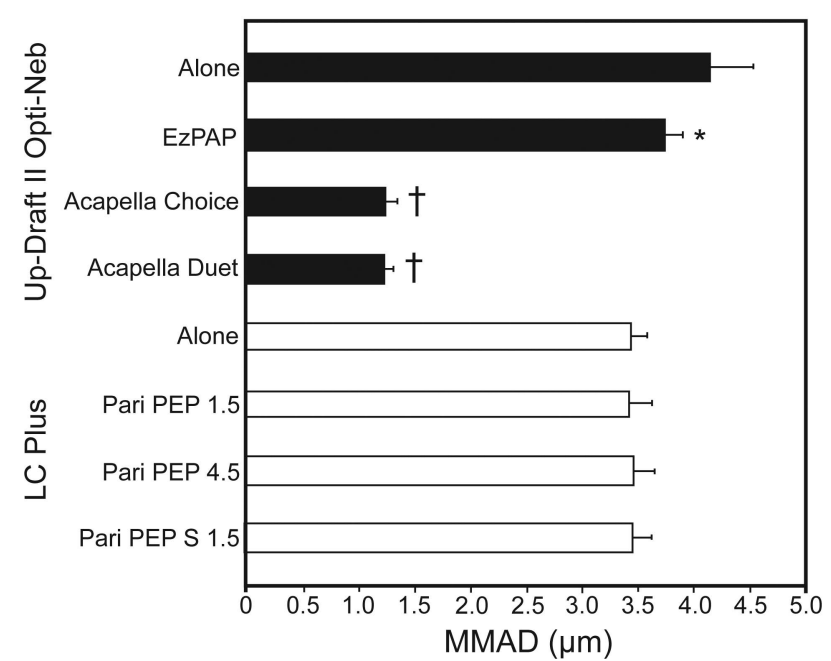

Fig. 5. Mass median aerodynamic diameter (MMAD) of aerosols from the Up-Draft II Opti-Neb and LC Plus nebulizers, alone and connected to PEP devices: Acapella Duet, Acapella Choice, EzPAP, Pari PEP, and Pari PEP S, with the resistance set at 1.5 or 4.5. The data bars represent the means from 6 experiments. The whisker bars represent the standard deviations. ${ }^{*} P=.02 \mathrm{com}-$ pared to nebulizer alone. $\dagger P<.001$ compared to nebulizer alone.

\section{Statistical Analysis}

Aerosol characteristics (MMAD, geometric standard deviation, particles $<5 \mu \mathrm{m}$, particles $1-3 \mu \mathrm{m}$, and mass balance), drug remaining in the nebulizer cup, and patient dose were compared with analysis of variance, followed by post-hoc analysis with the Dunnet test. Differences in patient dose among the breathing patterns were compared with analysis of variance, followed by the Tukey test for multiple comparison analysis. Statistical significance was set at .05. Statistics software was used for data analysis (Kaleidagraph 4.1, Synergy Software, Reading, Pennsylvania).

\section{Results}

\section{Particle Size Analysis}

The MMAD for the LC Plus alone was $3.42 \mu \mathrm{m} \pm$ $0.15 \mu \mathrm{m}$, and ranged from $3.42 \mu \mathrm{m}$ to $3.45 \mu \mathrm{m}$ when connected to the PEP devices $(P=.92)$ (Fig. 5, Table 1). The geometric standard deviation for the LC Plus alone was $2.25 \pm 0.04$, and ranged from 2.26 to 2.29 when connected to the PEP devices $(P=.31)$. The percentage of particles $<5 \mu \mathrm{m}$ was $66.9 \% \pm 2.1 \%$ for the LC Plus alone, and ranged from $66.6 \%$ to $66.8 \%$ when connected to the PEP devices $(P=.97)$. The percentage of particles between $1 \mu \mathrm{m}$ and $3 \mu \mathrm{m}$ for the LC Plus alone was $35.9 \% \pm 1.8 \%$, and ranged from $34.5 \%$ to $38.4 \%$ when connected to the PEP devices $(P=.11)$. The total albu- terol mass captured by the cascade impactor was $1,031 \pm 57 \mu \mathrm{g}$ for the LC Plus alone, and ranged from $1,105 \mu \mathrm{g}$ to $1,122 \mu \mathrm{g}(P=.01)$. The amount of albuterol that remained in the nebulizer cup was $1,375 \pm 30 \mu \mathrm{g}$ for the LC Plus alone, and ranged from $1,311 \mu \mathrm{g}$ to $1,372 \mu \mathrm{g}$ when connected to the PEP devices $(P=.11)$. The mass balance was similar among all devices $(P=.06)$.

The MMAD for the Up-Draft II Opti-Neb alone was $4.13 \mu \mathrm{m} \pm 0.4 \mu \mathrm{m}$, and decreased to $3.72 \mu \mathrm{m} \pm 0.18$, $1.24 \pm 0.1 \mu \mathrm{m}$, and $1.22 \pm 0.09 \mu \mathrm{m}$ when connected to $\operatorname{EzPAP}(P=.02)$, Acapella Choice $(P<.001)$, and Acapella Duet $(P<.001)$, respectively (see Fig. 5). The geometric standard deviation for the Up-Draft II Opti-Neb alone was $2.15 \pm 0.12$, and ranged from 1.89 to 3.12 when connected to the PEP devices $(P=.06)$. The percentage of particles $<5 \mu \mathrm{m}$ for the Up-Draft II Opti-Neb alone was $59.1 \% \pm 5.3 \%$, and remained unchanged when connected to EzPAP $(63 \%, P=.56)$. However, it increased to $86.5 \%$ and $89.1 \%$ when connected to Acapella Choice $(P<.001)$ and Acapella Duet $(P<.001)$, respectively. The percentage of particles between $1 \mu \mathrm{m}$ and $3 \mu \mathrm{m}$ for the Up-Draft II Opti-Neb alone was $25.1 \% \pm 2.9 \%$, and remained unchanged when connected to EzPAP (27.4\%, $P=.88)$. However, it increased to $46 \%$ and $44.3 \%$ when connected to Acapella Choice $(P<.001)$ and Acapella Duet $(P<.001)$, respectively (see Table 1$)$. The total albuterol mass captured by the cascade impactor was $570 \pm 126 \mu \mathrm{g}$ for the Up-Draft II Opti-Neb alone, remained unchanged with EzPAP $(P=.98)$, and decreased by $65 \%$ and $69 \%$ when connected to Acapella Choice $(P<.001)$ and Acapella Duet $(P<.001)$, respectively. Both the Acapella Choice and Acapella Duet retained a substantial amount of albuterol: $640 \pm 108 \mu \mathrm{g}$ and $419 \pm 230 \mu \mathrm{g}$, respectively. The amount of albuterol that remained in the nebulizer cup was $1,953 \pm 132 \mu \mathrm{g}$ for the Up-Draft II Opti-Neb alone, and ranged from 1,930 $\mu \mathrm{g}$ to $1,957 \mu \mathrm{g}$ when connected to the PEP devices $(P=.98)$. The mass balance was equal among all devices $(P=.25)$.

\section{Patient Dose}

There were no differences in patient dose between the LC Plus alone and the LC Plus connected to the PEP devices $(P=.05, P=.37$, and $P=.07$ for the older child, small adult, and large adult breathing patterns, respectively). Patient dose increased $(P<.001)$ with larger $\mathrm{V}_{\mathrm{T}}$ (Table 2).

The Up-Draft II Opti-Neb alone had a patient dose similar to that of the Up-Draft II Opti-Neb with EzPAP $(P=.99$, $P=.07$, and $P=.06$ for the older child, small adult, and large adult breathing patterns, respectively). However, connecting the Up-Draft II Opti-Neb to either the Acapella Choice or Acapella Duet decreased the patient dose by $76-84 \%$ with all 3 breathing patterns. $(P=.003$ and 


\section{In Vitro Evaluation of Positive Expiratory Pressure Devices Attached to Nebulizers}

Table 1. Characterization of Aerosols Generated by Nebulizers Alone and Connected to PEP Devices

\begin{tabular}{|c|c|c|c|c|c|c|c|c|}
\hline & \multicolumn{4}{|c|}{ Up-Draft II Opti-Neb } & \multicolumn{4}{|c|}{ LC Plus } \\
\hline & Alone & EzPAP & $\begin{array}{l}\text { Acapella Duet } \\
\text { (resistance } \\
\text { setting 1) }\end{array}$ & $\begin{array}{l}\text { Acapella Choice } \\
\text { (resistance } \\
\text { setting 1) }\end{array}$ & Alone & $\begin{array}{c}\text { Pari PEP } \\
\text { (resistance } \\
\text { setting 1.5) }\end{array}$ & $\begin{array}{c}\text { Pari PEP } \\
\text { (resistance } \\
\text { setting } 4.5 \text { ) }\end{array}$ & $\begin{array}{l}\text { Pari PEP S } \\
\text { (resistance } \\
\text { setting 1.5) }\end{array}$ \\
\hline $\begin{array}{l}\text { Mass median aerodynamic } \\
\text { diameter, } \mu \mathrm{m}\end{array}$ & $4.13 \pm 0.40$ & $3.72 \pm 0.18^{*}$ & $1.24 \pm 0.10 \dagger$ & $1.22 \pm 0.09 \dagger$ & $3.42 \pm 0.15$ & $5.42 \pm 0.20$ & $03.45 \pm 0.19$ & $3.44 \pm 0.17$ \\
\hline Geometric standard deviation & $2.15 \pm 0.12$ & $2.34 \pm 0.20$ & $1.89 \pm 0.05$ & $3.12 \pm 1.54$ & $2.25 \pm 0.04$ & $2.27 \pm 0.04$ & $42.29 \pm 0.11$ & $2.26 \pm 0.04$ \\
\hline $\begin{array}{l}\text { Percentage of aerosol particles } \\
\quad<5 \mu \mathrm{m}\end{array}$ & $59.1 \pm 5.3$ & $63.0 \pm 2.3$ & $86.5 \pm 6.2 \dagger$ & $89.1 \pm 8.2 \dagger$ & $66.9 \pm 2.1$ & $66.6 \pm 2.5$ & $66.8 \pm 2.8$ & $66.8 \pm 2.6$ \\
\hline $\begin{array}{l}\text { Percentage of aerosol particles } \\
1-3 \mu \mathrm{m}\end{array}$ & $25.1 \pm 2.9$ & $27.4 \pm 2.6$ & $46.0 \pm 8.6 \dagger$ & $44.3 \pm 9.4 \dagger$ & $35.9 \pm 1.8$ & $38.4 \pm 2.9$ & $37.4 \pm 2.5$ & $34.5 \pm 2.8$ \\
\hline $\begin{array}{l}\text { Total albuterol mass captured, } \\
\mu \mathrm{g}\end{array}$ & $570 \pm 126$ & $584 \pm 94$ & $176 \pm 26 \dagger$ & $200 \pm 68 \dagger$ & $1,031 \pm 57$ & $1,105 \pm 66$ & $1,116 \pm 62$ & $1,122 \pm 44$ \\
\hline $\begin{array}{l}\text { Reservoir, } \mu \mathrm{g} \\
\text { Device, } \mu \mathrm{g}\end{array}$ & $1,953 \pm 131$ & $1,930 \pm 189$ & $\begin{array}{r}1,957 \pm 162 \\
419 \pm 230\end{array}$ & $\begin{array}{r}1,936 \pm 165 \\
640 \pm 108\end{array}$ & $1,375 \pm 30$ & $1,372 \pm 85$ & $1,311 \pm 46$ & $1,357 \pm 64$ \\
\hline $\begin{array}{l}\text { Mass balance, } \mu \mathrm{g} \\
\text { Values are mean } \pm \mathrm{S} \mathrm{D} \text {. } \\
* P=.02 \text { compared to nebulizer alone. } \\
\dagger P<.001 \text { compared to nebulizer alone. } \\
\mathrm{PEP}=\text { positive expiratory pressure }\end{array}$ & $2,523 \pm 42$ & $2,514 \pm 117$ & $2,555 \pm 402$ & $2,773 \pm 254$ & $2,406 \pm 48$ & $2,478 \pm 102$ & $2,427 \pm 38$ & $2,479 \pm 37$ \\
\hline
\end{tabular}

Table 2. Patient Dose of Albuterol

\begin{tabular}{|c|c|c|c|c|}
\hline \multirow{2}{*}{ Nebulizer } & \multirow{2}{*}{ PEP Device } & \multicolumn{3}{|c|}{ Breathing Pattern } \\
\hline & & Child & Small Adult & Large Adult \\
\hline \multirow[t]{4}{*}{ Up-Draft II Opti-Neb } & None & $244 \pm 27$ & $272 \pm 25$ & $296 \pm 54$ \\
\hline & EzPAP & $240 \pm 71$ & $227 \pm 20$ & $198 \pm 43$ \\
\hline & Acapella Duet & $58 \pm 9 *$ & $51 \pm 11^{*}$ & $49 \pm 7 *$ \\
\hline & Acapella Choice & $49 \pm 8^{*}$ & $38 \pm 5^{*}$ & $34 \pm 8^{*}$ \\
\hline \multirow[t]{4}{*}{ LC Plus } & None & $338 \pm 6 \dagger$ & $455 \pm 24 \dagger$ & $530 \pm 18 \dagger$ \\
\hline & Pari PEP (resistance setting 1.5) & $302 \pm 13 \dagger$ & $478 \pm 9 \dagger$ & $558 \pm 14 \dagger$ \\
\hline & Pari PEP (resistance setting 4.5) & $349 \pm 18 \dagger$ & $493 \pm 21 \dagger$ & $537 \pm 19 \dagger$ \\
\hline & Pari PEP S (resistance setting 1.5) & $318 \pm 17 \dagger$ & $474 \pm 24 \dagger$ & $587 \pm 25 \dagger$ \\
\hline \multicolumn{5}{|c|}{$\begin{array}{l}\text { Values are mean } \pm \mathrm{SD} \mu \mathrm{g} \text {. } \\
* P<.001 \text { compared to nebulizer alone. } \\
\dagger P<.001 \text { compared to other breathing patterns. } \\
\text { PEP }=\text { positive expiratory pressure }\end{array}$} \\
\hline
\end{tabular}

$P=.003$ for the child pattern, $P<.001$ and $P<.001$ for the small adult pattern, and $P<.001$ and $P<.001$ for the large adult pattern, with Acapella Choice and Acapella Duet, respectively). There were no significant differences in patient dose between the different breathing patterns with any of the configurations.

\section{Discussion}

The tested PEP/vibratory PEP devices that obstruct the aerosol pathway decreased the aerosol particle size and lowered the patient dose produced by the Up-Draft II Opti-Neb. Larger $\mathrm{V}_{\mathrm{T}}$ increased patient dose with the LC Plus, but not with the Up-Draft II Opti-Neb.
The cascade impactor data for the LC Plus alone were similar to previously published work that used the same methodology. ${ }^{8}$ Laube et al compared the LC Plus with and without a PEP device and found a reduction in MMAD, from $4.07 \mu \mathrm{m}$ to $3.26 \mu \mathrm{m}$ (based on diethylene triamine pentaacetic acid [DTPA] measurement) when PEP was used. $^{6}$ They also reported that MMAD changed from $3.50 \mu \mathrm{m}$ to $2.82 \mu \mathrm{m}$ when the same experiments were done with albuterol sulfate. The different findings could be explained in part by several methodological differences between the studies. Laube et al used a Marple Miller impactor at $12 \mathrm{~L} / \mathrm{min}$ at room temperature, and I used Next Generation Impactor at $15 \mathrm{~L} / \mathrm{min}$, cooled to $4{ }^{\circ} \mathrm{C}$, therefore minimizing evaporation loss. I also used a 
different gas source (wall air vs compressor). In addition, Laube et al did not describe the PEP device or configuration they used, so it is possible that their model might have obstructed the aerosol pathway and thus increased impaction loss. In the in vivo part of their study, in which they used technetium ${ }^{99}$-DTPA aerosol, Laube et al found decreased pulmonary deposition and more peripheral deposition (larger inner/outer ratio) with the PEP device. However, when expressed as percentage of the loading dose, the addition of the PEP device decreased deposition in the inner area: inner deposition $2.7 \%$, outer deposition $2.4 \%$ without PEP, versus inner deposition $1.3 \%$, outer deposition $1.5 \%$ with PEP. Unfortunately, Laube et $\mathrm{al}^{6} \mathrm{did}$ not report other parameters, such as the percentages of particles $<5 \mu \mathrm{m}$ and 1-3 $\mu \mathrm{m}$. Those data could have helped to understand whether the changes in aerosol characteristics (especially on the smaller size fraction particles) was responsible for these findings. Other studies have shown significant differences in distribution of deposition between aerosols with MMAD of $1.01 \mu \mathrm{m}$ versus $3.68 \mu \mathrm{m} .{ }^{11}$

When the LC Plus was tested under breathing simulation conditions $\mathrm{I}$ found that larger $\mathrm{V}_{\mathrm{T}}$ produced larger patient dose. These data are in agreement with Barry et al, who, using similar technology, found that pediatric type breathing patterns $\left(\mathrm{V}_{\mathrm{T}} 150 \mathrm{~mL}\right)$ had lower patient dose than adult type breathing patterns $\left(\mathrm{V}_{\mathrm{T}} 600 \mathrm{~mL}\right) .{ }^{12}$

The type of PEP device used determined the changes that the aerosols experienced when connected to the Up-Draft II Opti-Neb. The changes in MMAD with the Acapella Choice and Acapella Duet $(4.32 \mu \mathrm{m}$ to $\sim 1.23 \mu \mathrm{m})$ are likely to alter the site of intrapulmonary deposition. ${ }^{5,11}$ In addition, there was a significant reduction in the dose captured by the cascade impactor (from 65\% to 69\%). Impaction loss within the PEP device is the most likely mechanism for that decrease: this was confirmed by the analysis of the washings from the PEP devices. The amount of aerosol that remained in the nebulizer was similar to nebulizer alone, so a decrease in aerosol generation was not responsible for the decrease in drug captured by the cascade impactor. The size selection resulting from the use of the Acapella with the Up-Draft II Opti-Neb is also evidenced by the increase in the percentages of particles $<5 \mu \mathrm{m}$ and particles 1-3 $\mu \mathrm{m}$. My findings are in agreement with Dornelas de Andrade, who found that the position of the nebulizer affected intrapulmonary deposition of a radio-labeled aerosol. ${ }^{7}$ They compared a nebulizer alone and placed before and after the Acapella device, and reported that placing the nebulizer after the Acapella significantly reduced lung deposition. More recently, Mitchell et al reported decreased albuterol mass (70\%) and MMAD when they coupled a breath-actuated nebulizer to the back of the Acapella device. ${ }^{13}$ They also reported that a proprietary PEP device that was coupled to the nebulizer without interrupting the aerosol pathway did not decrease either albuterol mass or MMAD. The EzPAP, which has the nebulizer placed between the PEP device and the mouthpiece, caused only a minor decrease in MMAD, which is unlikely to be clinically important. Moreover, the percentages of particles $<5 \mu \mathrm{m}$ and particles 1-3 $\mu \mathrm{m}$ were unchanged after adding the EzPAP.

My finding of a decrease in patient dose (by $76 \%$ and $84 \%$ ) when the nebulizer was connected to the Acapella device is in agreement with Mitchell et al, ${ }^{13}$ who also found that, similar to EzPAP, PEP devices that do not obstruct the aerosol pathway do not alter patient dose. The lack of increase in patient dose with larger $\mathrm{V}_{\mathrm{T}}$ seen with Acapella could be due to the inefficiency of aerosol delivery resulting from increased impaction. However, I have no clear explanation of why there were no changes with the nebulizer alone versus when connected to the EzPAP.

The ideal size of aerosol particles for patients with cystic fibrosis has been suggested by some authors to be between 2 and $3 \mu \mathrm{m},{ }^{14}$ but clinical trials have not provided conclusive evidence. ${ }^{14}$ The ideal size depends on where the aerosol is targeted and the inhalation technique. Drugs that target receptors in the large airways would benefit from larger particles. ${ }^{15}$ Laube et al reported a significant increase in intrapulmonary deposition in small and medium size aerosols when a slow inhalation maneuver was used. ${ }^{11}$

The limitations of this study are related to its in vitro nature and the methodology. The breathing simulation technique overestimates patient dose because it does not allow exhalation of particles, which occurs in human subjects. Also, although the use of idealized breathing patterns is widely accepted in aerosol research, it might not represent actual patient dose in vivo.

The clinical implications of these findings are important. The simultaneous use of nebulizer and PEP devices that obstruct the aerosol pathway may cause under-dosing, and waste drug. This might not be important with albuterol, but it is crucial for dornase alfa. In addition, the change of aerosol particle size could affect the deposition location and therefore increase systemic absorption and decrease the therapeutic response. ${ }^{15}$

\section{Conclusions}

In conclusion, simultaneous use of nebulizer and PEP devices that obstruct the aerosol pathway significantly decrease aerosol particle size and patient dose.

\section{REFERENCES}

1. O'Sullivan BP, Freedman SD. Cystic fibrosis. Lancet 2009; 373(9678):1891-904.

2. Mogayzel PJ Jr., Naureckas ET, Robinson KA, Mueller G, Hadjiliadis D, Hoag JB, et al. Cystic fibrosis pulmonary guidelines. Chronic medications for maintenance of lung health. Am J Respir Crit Care Med 2013 Apr 1;187(7):680-9. 


\section{In Vitro Evaluation of Positive Expiratory Pressure Devices Attached to Nebulizers}

3. Lester MK, Flume PA. Airway-clearance therapy guidelines and implementation. Respir Care 2009;54(6):733-50; discussion 751-3.

4. Smiths Medical. Respiratory care products brochure. http://www. smiths-medical.com/upload/products/pdf/Respiratory $\% 20$ care $\% 20$ brochure.pdf. Accessed on May 5, 2013.

5. Darquenne C. Aerosol deposition in health and disease. J Aerosol Med Pulm Drug Deliv 2012;25(3):140-7.

6. Laube BL, Geller DE, Lin TC, Dalby RN, Diener-West M, Zeitlin PL. Positive expiratory pressure changes aerosol distribution in patients with cystic fibrosis. Respir Care 2005;50(11):1438-44.

7. Dornelas de Andrade A, Mesquita F, Galindo Filho V, Menezes MJ, Ferreira Neto JL, Almeida Filho P, et al. Effects of the Acapella device in radioaerosol regional pulmonary deposition (abstract). Eur Respir J 2007;30:222.

8. Berlinski A, Hayden JB. Optimization of a procedure used to measure aerosol characteristics of nebulized solutions using a cooled next generation impactor. J Aerosol Med Pulm Drug Deliv 2010,23(6): 397-404.

9. 2.9.44 Preparations for nebulization: characterization. Pharmeuropa 2006; 18(2):280-283.
10. Aerosols, nasal sprays, metered dose inhalers and dry powder inhalers. US Pharmacopeia 28;2359-2377.

11. Laube BL, Jashnani R, Dalby RN, Zeitlin PL. Targeting aerosol deposition in patients with cystic fibrosis: effects of alterations in particle size and inspiratory flow rate. Chest 2000;118(4):1069-1076.

12. Barry PW, O'Callaghan C. An in vitro analysis of the output of salbutamol from different nebulizers. Eur Respir J 1999;13(5): 1164-1169.

13. Mitchell J, Avvakoumova V, Schneider H, Ali R, Nagel M. Combining inhalation by a breath-actuated nebulizer (BAN) with exhalation through an oscillating positive pressure device (OPEP) offers the potential for optimal combined therapy (abstract). Am J Respir Crit Care Med 2013;187:A4116.

14. Geller DE. The science of aerosol delivery in cystic fibrosis. Pediatr Pulmonol 2008;43:S5-S17.

15. Usmani OS, Biddiscombe MF, Nightingale JA, Underwood SR, Barnes PJ. Effects of bronchodilator particle size in asthmatic patients using monodisperse aerosols. J Appl Physiol 2003;95(5): 2106-2112. 